\title{
Unusual ferromagnetism in Ising and Potts model on semi-directed Barabási-Albert networks
}

\author{
Muneer A. Sumour ${ }^{1}$ and F. W. S. Lima ${ }^{2}$ \\ ${ }^{1}$ Physics Department, Al-Aqsa University, P.O.4051, Gaza, Gaza Strip, Pales- \\ tinian Authority, \\ ${ }^{2}$ Dietrich Stauffer Computational Physics Lab, Departamento de Física, \\ Universidade Federal do Piauí, 64049-550, Teresina - PI, Brazil. \\ e-mail: msumoor@alaqsa.edu.ps,fwslima@gmail.com
}

\begin{abstract}
We check the existence of a spontaneous magnetisation of Ising and Potts spins on semi-directed Barabasi-Albert networks by Monte Carlo simulations. We verified that the magnetisation for different temperatures $T$ decays after a characteristic time $\tau(T)$, which we extrapolate to diverge at positive temperatures $T_{c}(N)$ by a Vogel-Fulcher law, with $T_{c}(N)$ increasing logarithmically with network size $N$.
\end{abstract}

Keywords: Ising, Potts, Monte Carlo Simulations.

\section{Introduction}

The Ising and Potts model has been used during a long time as a "toy model" to test and to improve new algorithms and methods of high precision for calculation of critical exponents in Equilibrium Statistical Mechanics using the Monte Carlo method as Metropolis [1], Swendsen-Wang [2], Wang-Landau [3] algorithms. The Ising model was already applied to scale free networks [4] or undirected Barabási-Albert networks (UBA), where simulations [5] indicate a Curie temperature increasing logarithmically with increasing system size $N$. Different from [5], Sumour et al. [6, 7] studied the Ising model on a directed Barabási-Albert network (DBA) using standard Glauber kinetic Ising models on fixed networks. They confirmed the asymptoptic Arrhenius extrapolation $1 / \ln \tau \propto T$ for the time $\tau$ until the first sign change of the magnetisation, meaning that at all finite temperatures the magnetisation eventually vanishes, i.e., no ferromagnetism. 
In the present work, we study the critical behaviour of Ising and Potts model on semi-directed Barabási-Albert network (SDBA) recently studied by Sumour and Radwan [9], where now the number $N(k)$ of nodes with $k$ links each decays as $1 / k^{\gamma}$ and the exponent $\gamma$ decreases from 3 to 2 for increasing $m$ where $m$ is the number of old nodes which a new node added to the network selects to be connected with. This behaviour is totally different from $U B A$ and $D B A$ scale free networks where $\gamma=3$ is universal, i.e., independent of $m$. For both Ising and Potts model in our results no usual phase transition was found, similar to [5, 6, 7].

\section{Semi-Directed Barabási-Albert networks}

Both UBA and DBA networks are grown such that the probability, of a new site to be connected to one of the already existing sites, is proportional to the number of previous connections to this already existing site: the rich get richer. In this way, each new site selects exactly $m$ old sites as neighbours. In a UBA network [5], the neighbour relations for the spin interactions were such that if $\mathrm{A}$ has $\mathrm{B}$ as a neighbour, $\mathrm{B}$ has $\mathrm{A}$ as a neighbour, while for DBA in general $\mathrm{B}$ then does not have $\mathrm{A}$ as a neighbour.

In the DBA and UBA network [6, 7], if a new node selected $m$ old nodes as neighbours, then the $m$ old nodes are added to the Kertész list [8], and the new node is also added $m$ times to that list. Connections are made with $m$ randomly selected elements of that list. If one would only add the old nodes to the list, then only the initial core can be selected as neighbours, which is not interesting. But if one adds to the list the m old nodes plus once the new node, one has a semi-directed network: SDBA. (For usual BA networks, the new node is added $m$ times to the Kertész list.)

\section{Model and simulation}

\subsection{Two versions SDBA1 and SDBA2}

Our first version SDBA1 builds the network in the way of [9]. The new node $n$ selects $m$ sites $j$ which $n$ will all influence, while $n$ will be influenced only by the first selected $j$. Our second version SDBA2 inverts the direction of the spin interaction: The new node $n$ selects $m$ sites $j$ which will all influence $n$, while $n$ will influence only the first selected $j$. 
We simulate networks with $N$ nodes $i$, with spins $S_{i}$ on each node. For both Ising and Potts model on SDBA the evolution in time is given by single spin-flip Glauber dynamics with a probability $p$ given by

$$
p=1 /\left[1+\exp \left(2 \Delta E / k_{B} T\right)\right]
$$

with energy change $\Delta E$ to be defined below through eq. (2) and (3). Here, the time is defined as one Monte Carlo step (MCS), where one MCS is accomplished after all $N$ spins are updated; and we denote the final Monte Carlo step number as MCSN. (We use the same number of iterations for equilibration.) The error bars are usually smaller than the size of symbols, so we cannot put them into the figures. The statistical errors were evaluated from 10 to 100 samples of initial configurations and with 4,000 to 100,000 Monte Carlo steps ( thermal error).

\subsection{Ising model on SDBA networks}

The Ising interaction energy is given by

$$
E=-J \sum_{i} \sum_{j} S_{i} S_{j}
$$

where $S_{i}= \pm 1$ and the inner sum (also in eq. (3)) runs over all neighbours $j$ of node $i$. The magnetisation defined for this model is $\sum_{i=1}^{N} S_{i} / N$.

\subsection{Potts model on SDBA networks}

For Potts model the interaction energy is

$$
E=-J \sum_{i} \sum_{j} \delta_{S_{i} S_{j}}
$$

with Kronecker's delta and $S_{i}=1,2, \ldots q$. Again, to study the critical behaviour we define the magnetisation as $(q M-N) /((q-1) N)$, where $M$ is the largest of the $q$ numbers of spins $S_{i}$ in one of the $q$ directions $1,2, \ldots, q$, at each iteration. 


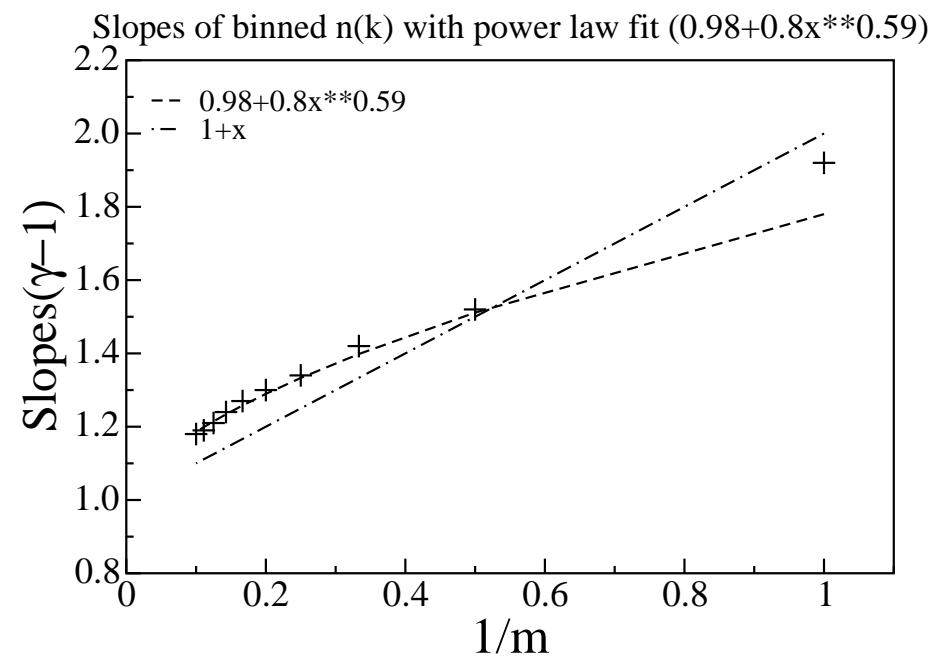

Figure 1: Plot of $\gamma(m)-1$ versus $1 / m$ with power-law : $\gamma-1=0.98+$ $0.8 / m^{0.59} ; N=400,100,000$ nodes.

\section{$4 \quad$ Results and discussion}

\subsection{Ising model}

We use the FORTRAN program as in our appendix (1), with different $m$. The number of nodes $N$ added to the initial core of $m$ nodes is 10000 to 50000 , and MCSN $=100,000$ iterations were made. First we measure the number $N(k)$ of nodes influenced by $k$ neighbours in SDBA2, analogous to [9] for SDBA1. In Fig. 1 we plot for each $m$ value (including $m=1$, not shown) we plotted double-logarithmically the observed numbers of nodes with at least $k$ links each and determined the decay exponents by the slopes $\gamma(m)-1$ versus $1 / m$, which makes clearer the possible extrapolation towards infinite $m(m=\infty, 1 / m=0)$. Maybe the true exponents $\gamma(m)$ equal $2+1 / m$ since $m=1$ should give the standard (undirected) exponent $\gamma=3$. The deviations from this formula (straight line in Fig. 11) are not much larger than our systematic errors. As an alternative to the linear behaviour also a power-law fit to $m>1$ is shown. We see that the new power-law fit agrees very nicely with the data except for the standard BA model (undirected) case $m=1$. The behaviour of the exponents for much larger $m$ is discussed elsewhere [10] and differs appreciably between SDBA1 and SDBA2. Fig. 2 shows the 
Ising model on SDBA2 network, $\mathrm{m}=3, \mathrm{MCSN}=100,000$

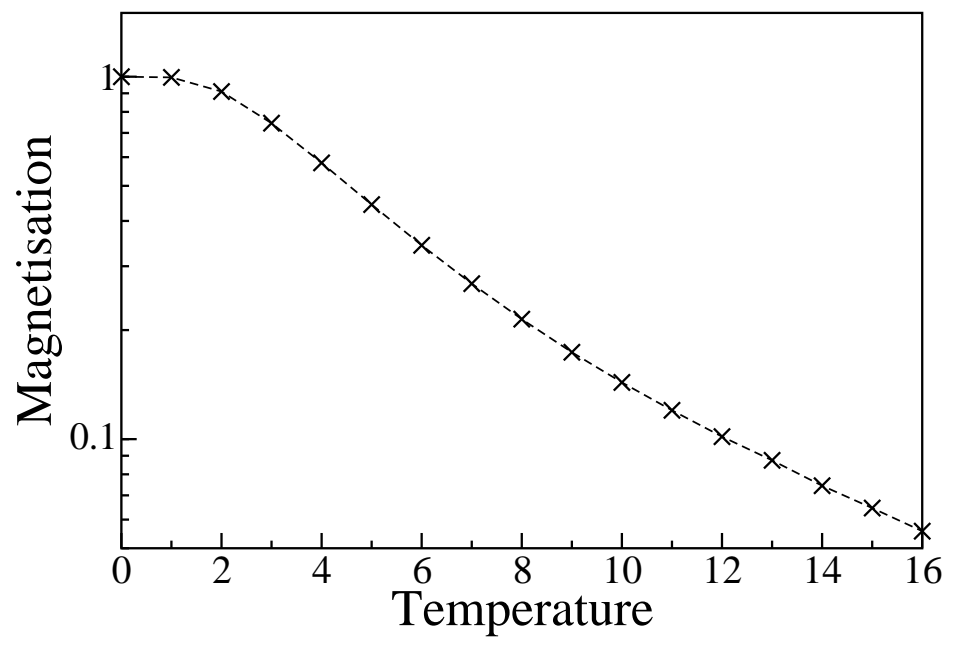

Figure 2: Semi-logarithmic plot of magnetisation versus $T$ for $m=3$ and 4000 nodes

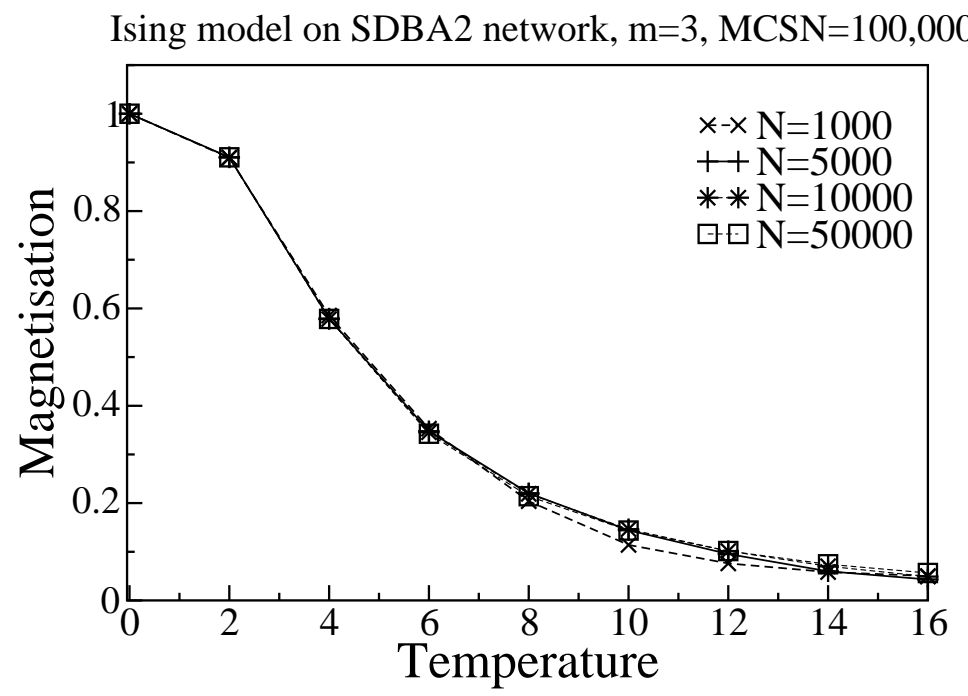

Figure 3: Plot of the magnetisation versus $T$ for $m=3$ and different system sizes $N=1000$ to $50,000, M C S N=100,000$. 

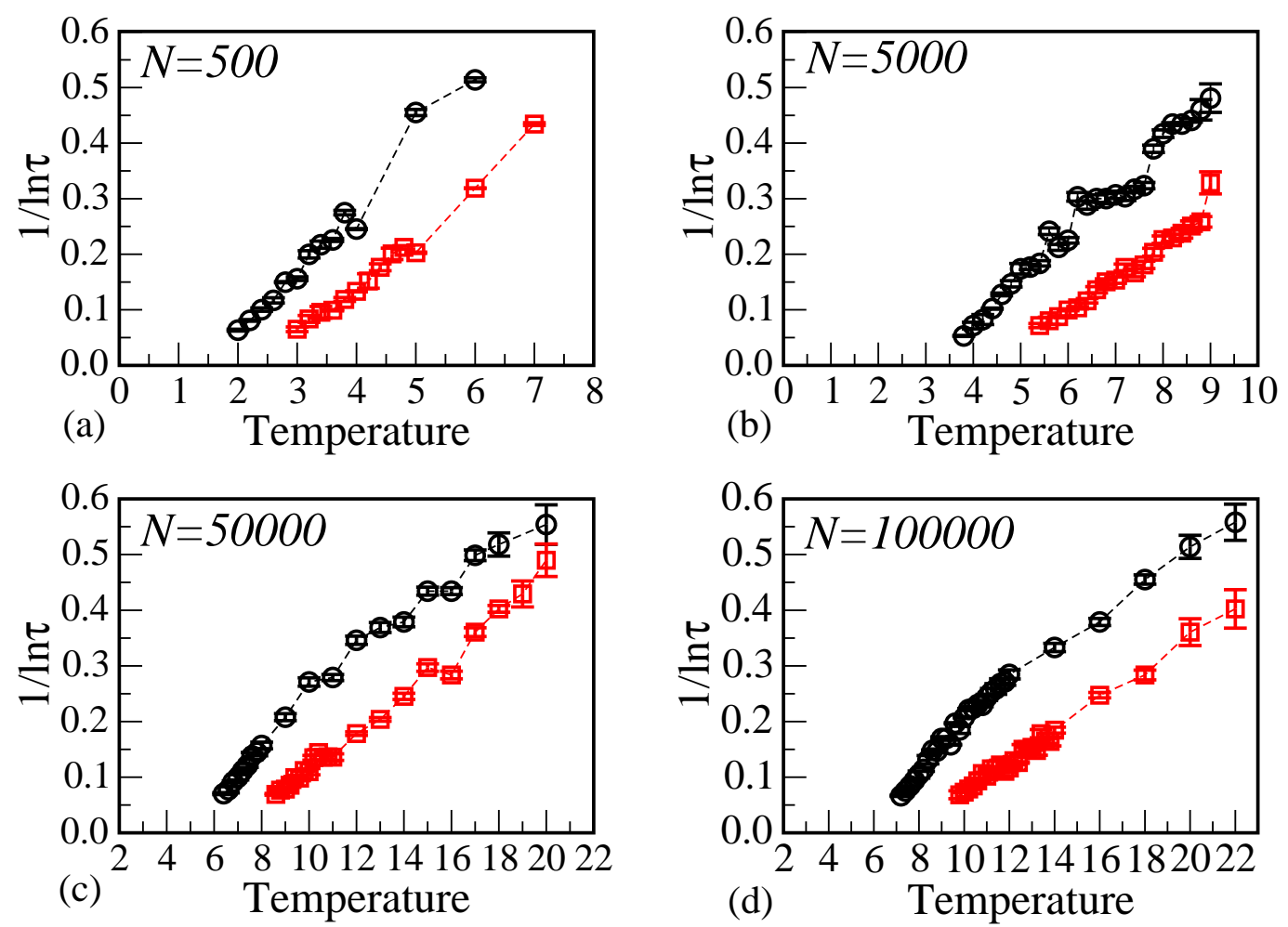

Figure 4: Reciprocal logarithm of the relaxation times versus temperature for SDBA1(circle) and SDBA2 (square) networks and Potts model with $q=2$ (Ising), $m=2$ initial neighbours and $N=500$ (a), 5000 (b), 50000 (c), and $100000(d)$ sites.

magnetisation as a function of temperature $(T=0,1,2, \ldots, 16)$. The roughly exponential decay is similar to [5]. Then we change the initial number of neighbours $m=1,3,5,7$ with system size 50000 and $M C S N=4,000$ to 100, 000 iterations.

\subsection{Potts model}

To study the $q=2$ Potts model we start with all spins ordered $S=1$, a number of spins equal to $N=500,5000,50000$, and 100000 with MCSN 
Potts model with $\mathrm{q}=2$ on SDBA1(x), SDBA2(+), M=3, N=50000

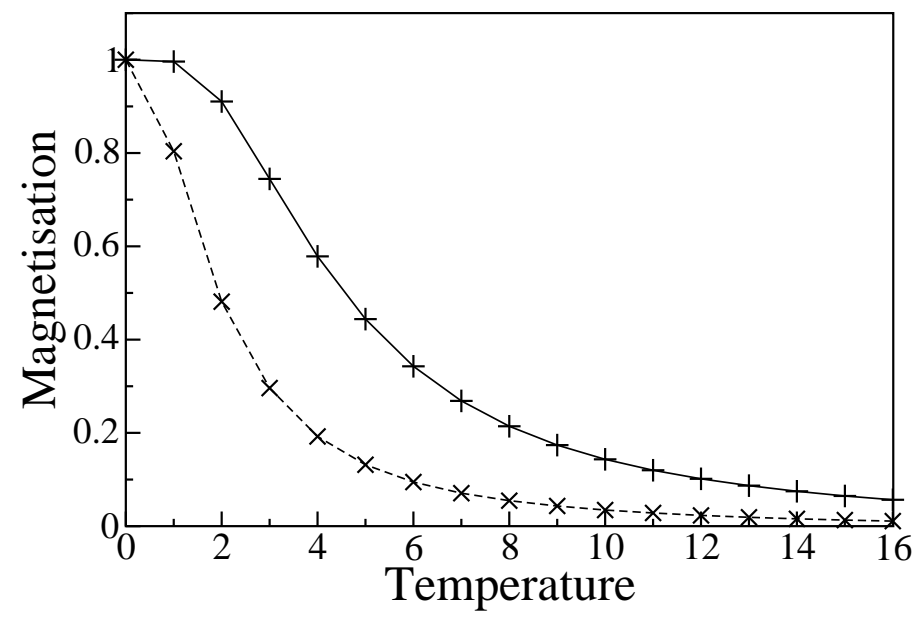

Figure 5: Plot of the magnetisation versus temperature on $\operatorname{SDBA} 1(x)$ and SDBA2(+) network for Potts model with $q=2$ states, $m=3$ initial neighbours, $N=50000$ sites and $M C S N=100,000$ iterations.

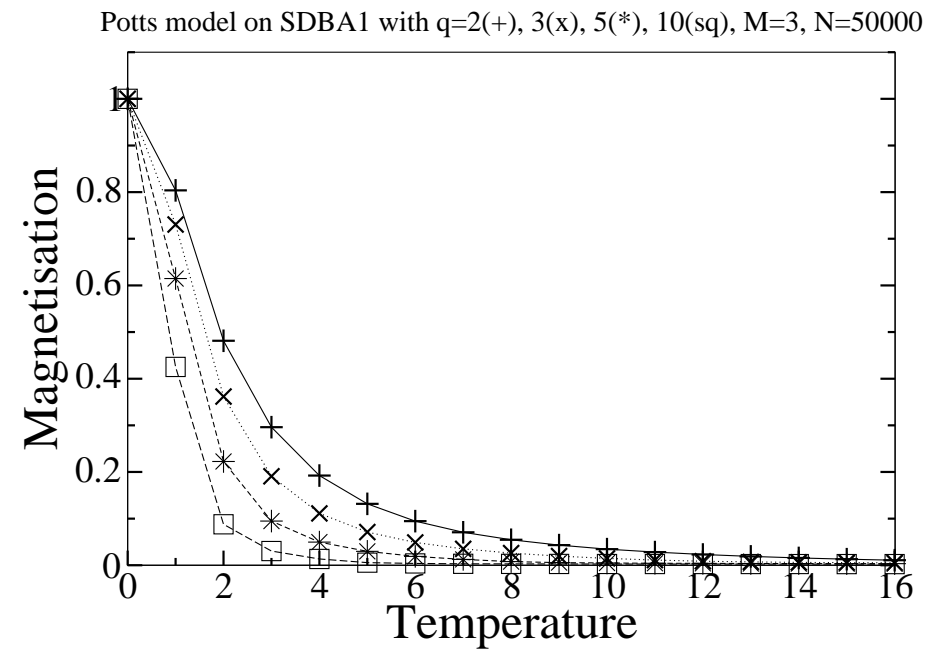

Figure 6: Plot of the magnetisation versus $T$ for different values of $q=$ $2(+), 3(x), 5(*)$, and 10(square) on SDBA1 network for $N=50000$ sites and $m=3$. 


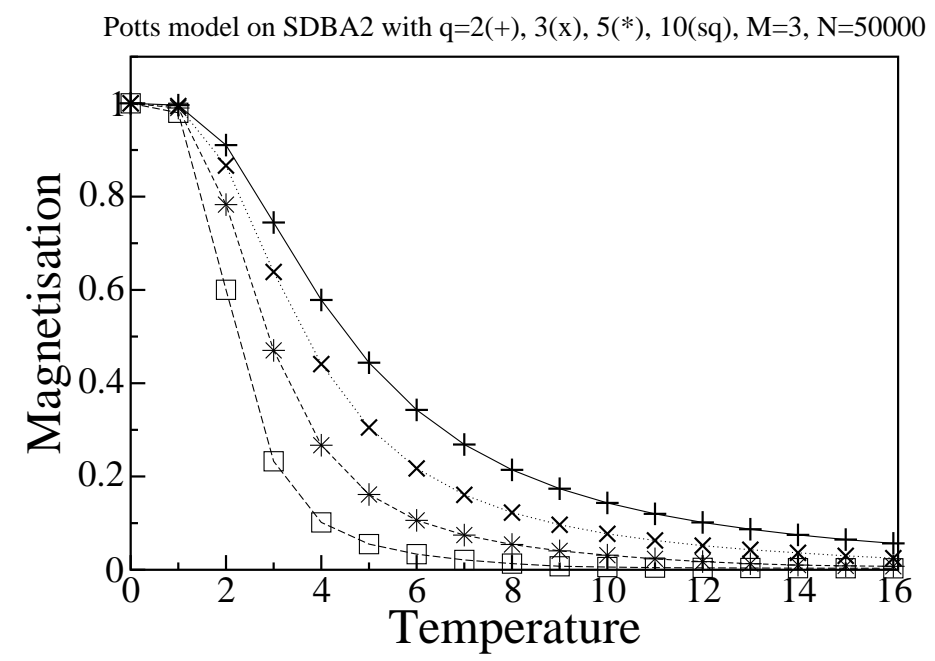

Figure 7: As previous figure but now for SDBA2.

$=10^{8}, 10^{7}$ and $2 \times 10^{6}$ with HeatBath algorithm, respectively, in Figs. 4 (a), (b), (c), and (d). The temperature is measured in units of $J / k_{B}$. We determine the time $\tau$ after which the magnetisation has flipped its sign for first time, and then take the median value of 9 samples. So this way is possible to determine various temperature for different networks size and to extrapolating $\tau$ to infinity and obtain the critical temperature for SDBA1 and SDBA2 networks . Our simulations on SDBA1 and SDBA2 networks

indicate that the $q=2$ Potts model does not display a usual phase transition and the plots of the time $1 / \ln (\tau)$ versus temperature in Figs. 4(a), (b), (c), and (d) show that our results agree with a Vogel-Fulcher(-Tammann) law for the relaxation time $\tau$, defined as the first time when the sign of the magnetisation flips: $1 / \ln (\tau) \propto T-T_{c}(N)$.

We extrapolate $T_{c}(N)$ for $N=20,30,50,100,200,500,5000,50000$, and 100000 to $\approx 0.1,0.2,0.7,1.0,1.1,1.3,3.1,5.3$, and 6.0 for SDBA1 and to $0.5,0.7,1.0,1.5,1.8,2.5,4.2,6.6$ and 7.0 for SDBA2, increasing roughly logarithmically with $N$ as in [5].

In Fig. 5 we show the magnetisation versus temperature behaviour on SDBA1 and SDBA2 network for Potts model with $q=2$ states and $m=3$ initial neighbours and $N=50000$ sites. Both SDBA1 and SDBA2 network present similar behaviour, but SDBA1 decreases faster than SDBA2 with 


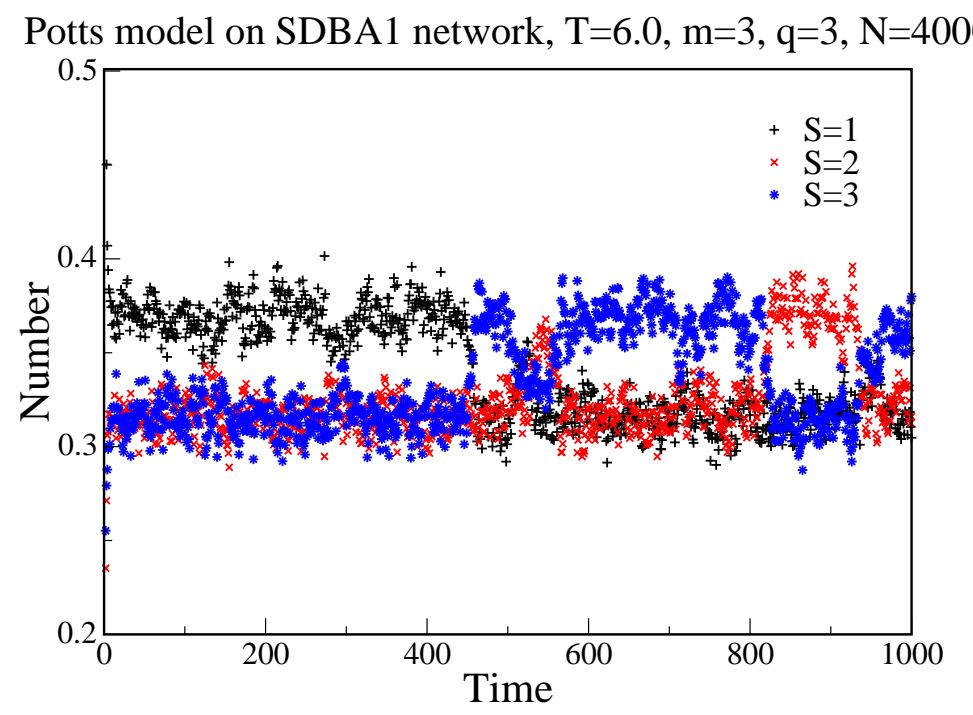

Figure 8: Plot of the number of $S=1,2$, and 3 states versus the time for Potts model with $q=3$ states on SDB1 network, with $m=3, N=4000$.

Potts model on SDBA2 network, $\mathrm{T}=9.0, \mathrm{~m}=3, \mathrm{q}=3, \mathrm{~N}=4000$

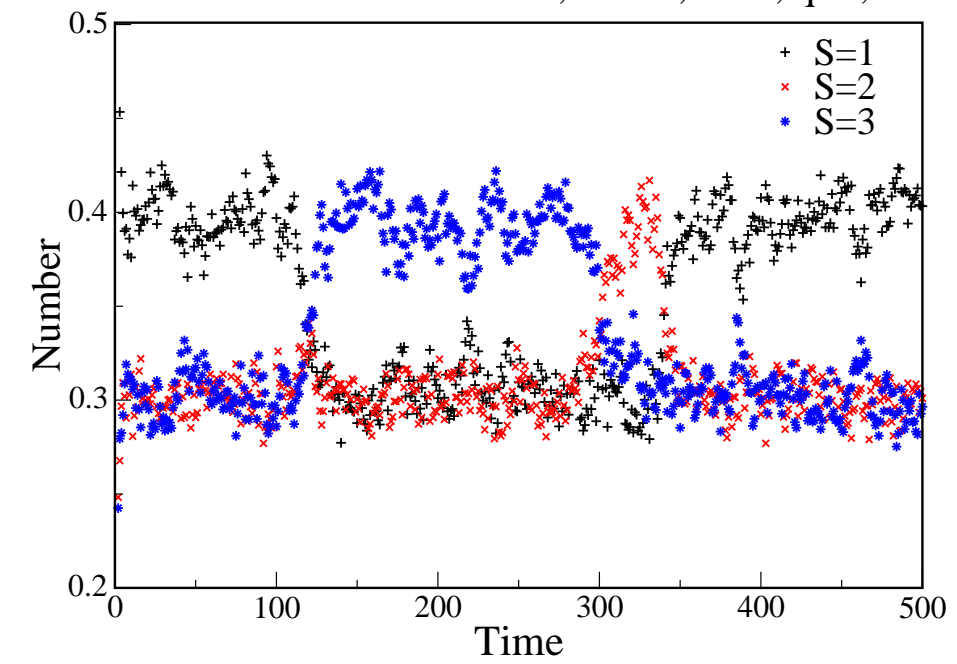

Figure 9: The same plot of Fig. 8, but now for SDBA2 networks. 
increasing temperature, since SDBA2 has more neighbours than SDBA1. We use two different programs for Potts and Ising which agreed in their results for $q=2$, and they should.

In Fig. 6] we show magnetisation versus temperature on SDBA1 network for Potts model with $q=2$, 3, and 10 states, $m=3$ initial neighbours and $N=50000$ sites. Here, we see that increasing $q$ of Potts model provides a more rapid decay of the magnetisation as a function of temperature. In Fig. 7 we show the same behaviour, but now on SDBA2 network.

In figure 8 we show the time dependence of the number of $S=1$ and 3 states for Potts model with $q=3$ states on SDBA1 network. Here we observe the tunneling between these three states with the evolution of time. In Fig. 9 we show the same behaviour as in Fig. 8, but now on SDBA2 network.

\section{Conclusion}

Finally, for both SDBA1 and SDBA2 networks we found a Vogel-Fulcher law, suggesting stable ferromagnetism for $T<T_{c}(N)$. For Potts model with $q=3$ on SDBA1 and SDBA2 networks we found a tunneling between these three states with the evolution of time. Similarly to the Ising model on undirected Barabási-Albert network [5] there is no usual ferromagnetic transition on these SDBA1 and SDBA2 networks, since the curves of magnetisation versus temperature are not curved in the usual way at least for $q$ up to 10, and the time dependence in the Ising case $(q=2)$ suggests a transition temperature increasing logarithmically with increasing system size, i.e., $T_{c}(N)$ increases roughly logarithmically with network size $N$. Perhaps the limit $q \rightarrow \infty$ would show a more usual behaviour as Fig. 6 and 7 allow. The distribution of the number of neighbours of each node decays with a non-universal exponent depending on $m$, as in 9 .

The authors are grateful to Dietrich Stauffer for stimulating discussions and for a critical reading of the manuscript. F. W. S. L. acknowledges the Brazilian agency FAPEPI (Teresina-Piauí-Brasil) and CNPQ for its financial

support and. This work also was supported the system SGI Altix 1350 the computational park CENAPAD.UNICAMP-USP, SP-BRAZIL and Dietrich Stauffer Computational Physics Lab-TERESINA-PIAUÍ-BRAZIL. 


\section{References}

[1] N. Metropolis, A. W. Rosenbluth, M. N. Rosenbluth, A. H. Teller, E. Teller, J. Chem. Phys. 21, 1087 (1953).

[2] R. H. Swendsen, J.-S. Wang, Phys. Rev. Lett. 58, 86 (1987).

[3] F. Wang, D. P. Landau, Phys. Rev. Lett. 86, 2050 (2001).

[4] A.-L. Barabási, R. Albert, Science 286, 509 (1999).

[5] A. Aleksiejuk, J.A. Hołyst, D. Stauffer, Physica A 310, 260 (2002) .

[6] M. A. Sumour, M.M. Shabat, Int. J. Mod. Phys. C 16, 585 (2005).

[7] M. A. Sumour, M.M. Shabat, D. Stauffer, Islamic University Journal (Gaza) 14, 209 (2006). cond-mat/0504460 at www.arXiv.org.

[8] D. Stauffer, S. Moss de Oliveira, P.M.C de Oliveira and J.S.Sá Martins, Biology, Sociology, Geology by Computational Physicists. Elsevier, Amsterdam (2006).

[9] M. A. Sumour and M. A. Radwan, Int. J. Mod. Phys. C 23, article 1250062 (2012).

[10] M. A. Sumour, F. W. S. Lima, M. A. Radwan, and M.M. Shabat, Islamic University Journal (Gaza), to be published. 


\section{Appendix}

This is the Fortran program for Ising model on SDBA2. For SDBA1 a program is given in [9], without spins.

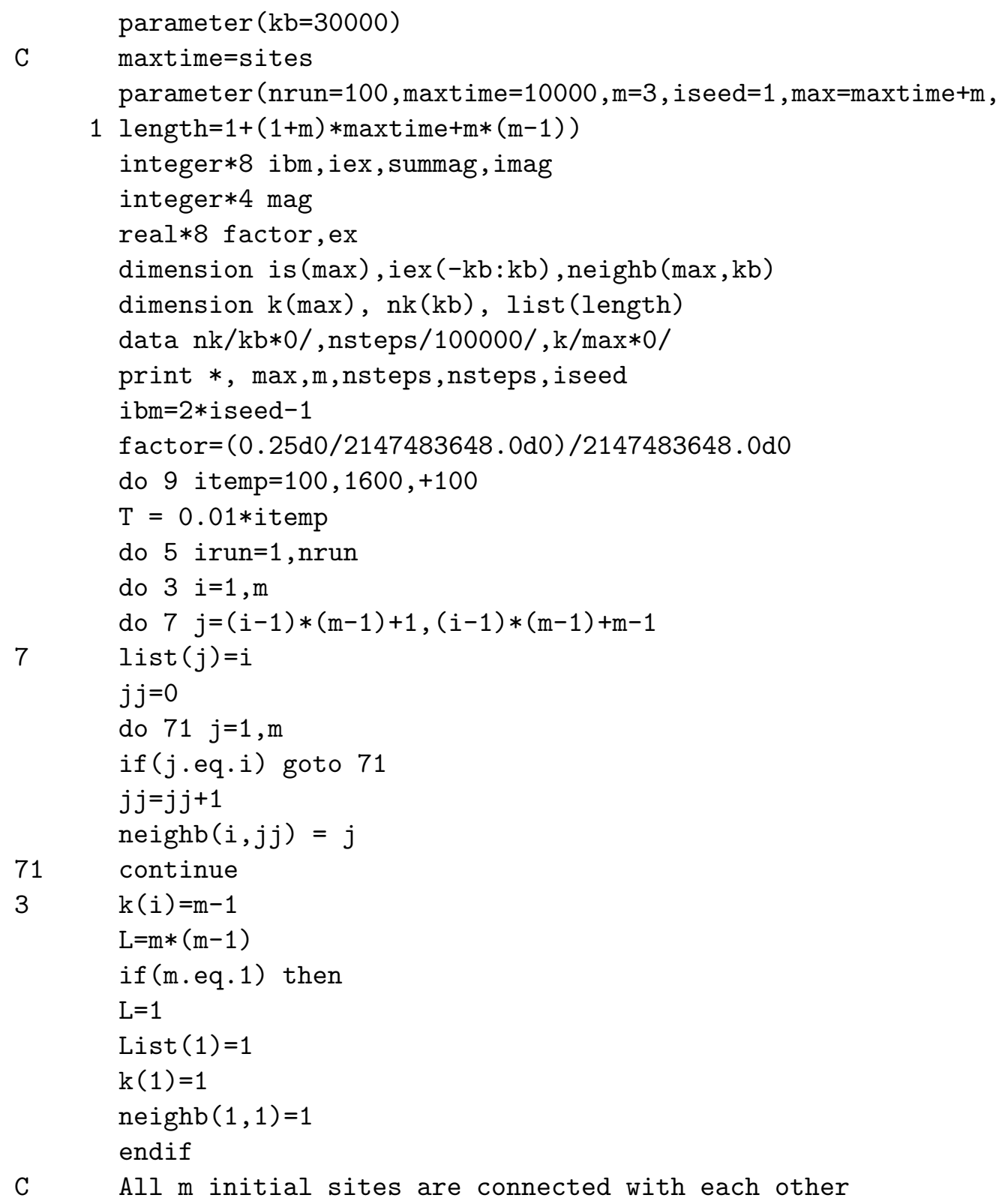




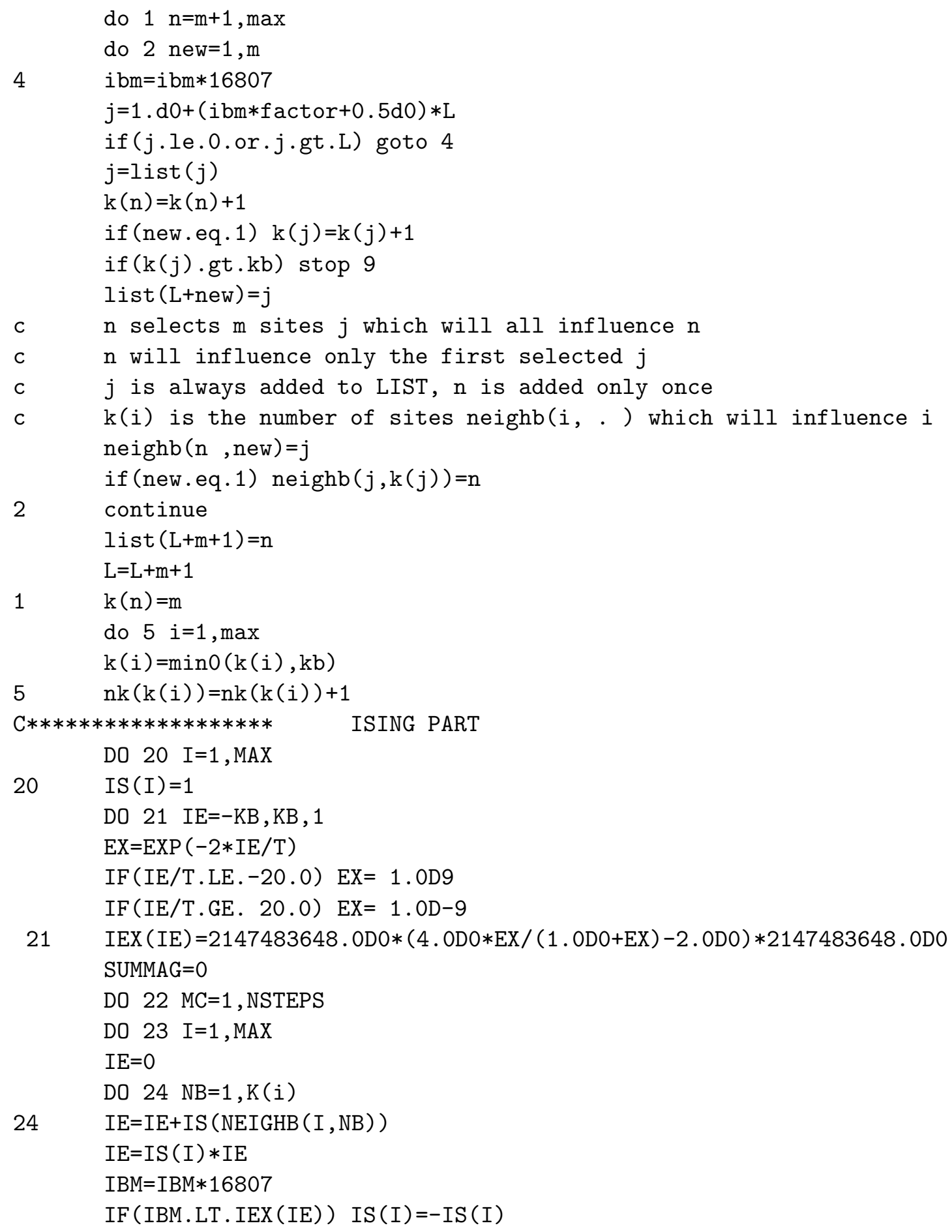


23 CONTINUE

MAG $=0$

DO $25 \mathrm{I}=1, \mathrm{MAX}$

$25 \quad M A G=M A G+I S(i)$

IMAG $=$ IABS $(M A G)$

22 IF (MC.GT. (NSTEPS/2)) SUMMAG=SUMMAG+IMAG

AVERGESUMMAG=SUMMAG $* 2.0 /($ MAX $*$ NSTEPS $)$

$C$ End of Ising part

9 PRINT *, T, AVERGESUMMAG

STOP

END 